\title{
THE APPROACH OF SOLUTIONS OF NONLINEAR DIFFUSION EQUATIONS TO TRAVELLING WAVE SOLUTIONS
}

\author{
BY PAUL C. FIFE AND J. B. McLEOD ${ }^{1}$
}

Communicated by Hans Weinberger, May 5, 1975

1. This note is concerned with the pure initial-value problem for the nonlinear diffusion equation

$$
u_{t}-u_{x x}-f(u)=0 \quad(-\infty<x<\infty, t>0)
$$

with $u(x, 0)=\phi(x)$. This problem has attracted an increasing amount of attention in recent years, one of the central questions being whether or not the solution $u(x, t)$ tends as $t \rightarrow \infty$ to a travelling wave solution $U(x-c t)$. ([1] gives a general bibliography.) We adopt the usual normalization of the problem by assuming throughout that $f \in C^{1}[0,1], f(0)=f(1)=0,0 \leqslant \phi \leqslant 1$, so that, as is well known, $0 \leqslant u(x, t) \leqslant 1$ for all $x, t$.

2. A typical convergence result that we can prove is the following.

TheOREM A. Let $f \in C^{1}[0,1]$, with $f(0)=f(1)=0, f^{\prime}(0)<0, f(1)<0$,

$$
f(u)<0 \text { for } 0<u<\alpha_{0}, \quad f(u)>0 \text { for } \alpha_{1}<u<1,
$$

and assume that there exists a travelling wave solution $U(x-c t)$ with $U(-\infty)=$ $1, U(\infty)=0,0 \leqslant U \leqslant 1$. Let $\phi$ satisfy $0 \leqslant \phi \leqslant 1, \lim _{\inf _{x \rightarrow-\infty}} \phi(x)>\alpha_{1}$, $\lim \sup _{x \rightarrow \infty} \phi(x)<\alpha_{0}$. Then there exists some $x_{0}$ such that,

$$
\lim _{t \rightarrow \infty}\left|u(x, t)-U\left(x-c t-x_{0}\right)\right|=0
$$

uniformly in $x$. If $\phi$ is monotonic, then the approach is in fact exponential.

We remark that such a travelling wave $U$ can be shown to be necessarily monotonic, and it is an obvious consequence of Theorem $\mathrm{A}$ that $U$ is unique up to translation. This can, of course, be shown directly (Theorem $\mathrm{C}$ below), and conditions under which $U$ will exist are discussed in Theorem $\mathrm{D}$.

In some cases the solution develops into a pair of diverging travelling waves, and this is relevent to the case where $\phi$ is of compact support.

THEOREM B. Let $f$ satisfy the hypotheses of Theorem A, and suppose $c>$

AMS (MOS) subject classifications (1970). Primary 35K15.

1 Research supported by a grant from the United Kingdom Science Research Council and U.S. Army Contract No. DA-31-124-ARO-D-462. 
0 . Let $\phi$ satisfy $0 \leqslant \phi \leqslant 1$, lim $\sup _{x \rightarrow \pm \infty} \phi(x)<\alpha_{0}$, and $\phi(x)>\alpha_{1}+\eta$ for some $\eta>0$ and a sufficiently long $x$-interval. Then there exists some $x_{0}$ and $x_{1}$ such that, uniformly in $x$,

$$
\lim _{t \rightarrow \infty}\left|u(x, t)-U\left(x-c t-x_{0}\right)-U\left(-x-c t-x_{1}\right)+1\right|=0 .
$$

We remark that the condition $c>0$ is equivalent to $\int_{0}^{1} f d u>0$.

To prove Theorems A and B, we write the solution as a function $u=$ $u^{*}(z, t)$, where $z=x-c t$. A comparison technique based on the maximum principle is used to obtain information about $u^{*}$ as $z, t \rightarrow \infty$ and to conclude that the set $\left\{u^{*}(\cdot, t), t \geqslant \delta>0\right\}$ is relatively compact in $C^{3}(-\infty, \infty)$. A Lyapunov functional is then used to show that the limit set consists of just one travelling wave solution.

3. If the initial value $\phi$ is monotonic, then it is standard that $u$ remains monotonic in $x$ for all $t$. Hence, we can change to $u, t$ as independent variables, with $v=u_{x}$ as the dependent variable. Differentiating (1) with respect to $x$, we obtain the corresponding problem for $v$ :

$$
v_{t}-v^{2} v_{u u}+f v_{u}-f_{u} v=0 \quad(0<u<1, t>0),
$$

with

$$
v(0, t)=0, \quad v(1, t)=0, \quad v(u, 0)=\Phi(u) .
$$

A travelling wave solution of (1) is a steady solution of (2), and we are interested in solutions for which $u(x, t)$ is monotonic decreasing in $x$, so that $v(u, t)<0$ for $u$ in $(0,1)$.

THEOREM C. If $f \in C^{1}[0,1]$, with $f(0)=f(1)=0$, and if $f(u) \leqslant 0$ for $u$ sufficiently small, while $f(u) \geqslant 0$ for $u$ sufficiently near 1 , then there is at most one steady solution of (2) satisfying $v(0)=v(1)=0, v<0$ in $(0,1)$.

The steady form of (2) integrates to give $v_{u}+f / v=$ constant $=-c$, say, $c$ ' being in fact the wave-speed. Theorem $\mathrm{C}$ is proved by showing that there is a monotonic dependence of $v$ on $c$, and this monotonicity is also used to discuss existence of steady solutions. If $f$ has just one interior zero in $(0,1)$, then there does exist a (unique) steady negative solution (with zero boundary data) over $[0,1]$, and there is associated with this a characteristic wave-speed. If $f$ has more interior zeros, the situation is more complicated.

THEOREM D. Suppose that $[0,1]$ is divided into $p$ subintervals $\left[u_{0}, u_{2}\right]$, $\left[u_{2}, u_{4}\right], \ldots,\left[u_{2 p-2}, u_{2 p}\right]$, where $u_{0}=0, u_{2 p}=1$, and that in each subinterval $\left(u_{2 r}, u_{2 r+2}\right)$ there exists a point $u_{2 r+1}$ such that either

$$
f \leqslant 0 \text { in }\left(u_{2 r}, u_{2 r+1}\right), f>0 \text { in }\left(u_{2 r+1}, u_{2 r+2}\right), \int_{u_{2 r}}^{u_{2 r+2} f d u>0,}
$$




$$
f<0 \text { in }\left(u_{2 r}, u_{2 r+1}\right), f \geqslant 0 \quad \text { in }\left(u_{2 r+1}, u_{2 r+2}\right), \int_{u_{2 r}}^{u_{2 r+2} f d u<0,}
$$

or

$$
f<0 \text { in }\left(u_{2 r}, u_{2 r+1}\right), f>0 \text { in }\left(u_{2 r+1}, u_{2 r+2}\right) \text {. }
$$

Then there exists a subset of $\left\{u_{2 r}\right\}$, say $\left\{U_{i}\right\}, i=0, \ldots, k$, with $U_{0}=0$, $U_{k}=1$, such that there is a (unique) steady negative solution of (2) (with zero boundary data) over $\left[U_{i}, U_{i+1}\right]$, but not over any $\left[u_{2 r}, u_{2 s}\right]$ unless it is a subinterval of some $\left[U_{i}, U_{i+1}\right]$. Further, if $c_{i}$ is the wave-speed associated with $\left[U_{i}, U_{i+1}\right]$, then $c_{i} \geqslant c_{i+1}$.

The physical interpretation of this is that the travelling waves corresponding to the subintervals $\left[u_{2 r}, u_{2 r+2}\right]$ of any $\left[U_{i}, U_{i+1}\right]$ have merged into a single travelling wave, but the travelling wave over $\left[U_{i}, U_{i+1}\right]$ is faster than that over $\left[U_{i+1}\right.$, $U_{i+2}$ ], since $c_{i} \geqslant c_{i+1}$, so that the two are moving apart (or at least not closing) and no single travelling wave can embrace them both.

By applying the maximum principle and ideas of sub- and super-solutions to the problem (2)-(3), we obtain

THEOREM E. If $f$ satisfies the conditions of Theorem $\mathrm{D}$, and $\Phi<0$ in $(0,1)$, then the solution of (2)-(3) converges uniformly in each $\left[U_{i}, U_{i+1}\right]$ as $t \rightarrow \infty$ to the steady negative solution (with zero boundary data) over $\left[U_{i}, U_{i+1}\right]$.

This theorem can be interpreted with $x$ and $t$ as independent variables and leads to a result comparable with Theorem A.

\section{REFERENCES}

1. D. G. Aronson and H. F. Weinberger, Nonlinear diffusion in population genetics, combustion, and nerve propagation, Proc. Tulane Program in Partial Differential Equations, Lecture Notes in Math. No. 446, Springer-Verlag, New York, 1975 (to appear).

MATHEMATICAL INSTITUTE, UNIVERSITY OF OXFORD, OXFORD, ENGLAND

DEPARTMENT OF MATHEMATICS, UNIVERSITY OF ARIZONA, TUCSON, ARIZONA 85721

MATHEMATICS RESEARCH CENTER, UNIVERSITY OF WISCONSIN-MADISON, MADISON, WISCONSIN 53706 